\title{
Controlling and Pricing Shareability
}

\author{
Thomas A. Weber \\ École Polytechnique Fédérale de Lausanne \\ thomas.weber@epfl.ch
}

\begin{abstract}
In the presence of a peer-to-peer economy, the option of sharing an item is valuable for consumers. By retaining control over the shareability of its products a monopolist can set a sharing tariff in conjunction with the purchase price of the product, in order to extract state-contingent surplus from consumers: the shareability rent. Using an overlapping-generations model with heterogeneous consumers, we determine the jointly optimal retail price and sharing tariff for durable products, and quantify the value for the control of shareability, thus defining the firm's financial boundary conditions for an investment in sharing-control technologies.
\end{abstract}

\section{Introduction}

The degree to which software, movie subscriptions, transportation tickets, and physical devices are shared among peers can in principle be controlled by the producer or a seller of these items. For example, in order to authorize an owner of an annual rail pass to share it with another person, the ticket issuer can ask for a fee, referred to as a "sharing tariff." Similarly, for computer games delivered and accessed through online portals (e.g., Steam), ownership for a game could be shared conditional on seller authorization. Even for physical devices, such as audio studio gear (e.g., by Antelope or United Audio), online registration may be required to gain access, which ultimately allows the producer to retain some control over peer-to-peer sharing and transfer of ownership. The limits of the control over the use of an item are due mainly to budget constraints or lack of imagination, rather than an intrinsic lack of feasibility ${ }^{1}$

We consider here the question of how to jointly set the product price and the sharing tariff in the presence of a costless peer-to-peer exchange. This allows us to quantify the value the company can extract by retaining control over the shareability of its products, which implicitly defines the budget constraint for the technology used to monitor sharing and collect the sharing tariff. The dynamic model we use is based on recent work by Weber (2016a) which features a dynamic sharing economy with overlapping generations of consumers (agents). The agents are heterogeneous with respect to the subjective likelihood of experiencing a need in any given period, and with respect to the "contingent" value for using the item when needed. The overlapping generations provide for the coexistence of agents in different phases of their consumption lifecycle: some consumers (in an early consumption phase) decide about ownership, while others (in a late consumption phase), having observed their needs, choose whether or not to participate in a sharing market.

\subsection{Literature}

The control of the shareability of products in a secondary market is reminiscent of market and aftermarket control through compatibility and technological complements. Manufacturers can try to limit the interoperability of devices in an attempt to limit access to a network and achieve customer lock-in to proprietary systems (Adams and Brock 1982; Farrell and Saloner 1992). Other options include tying the sale of one product to the purchase of another product (Carlton and Waldman 2002) or controlling technological complements, e.g., by requiring the use of proprietary cartridges with printers (Schulz 2015). When controlling the shareability of an item, a producer effectively asks for the purchase of a 'license to share' (i.e., a proprietary product) whenever the owner would like to 'augment' the use of his item by lending it out to others.

Benkler (2004) defines shareable goods as (i) technically lumpy, i.e., they provide "functionality in discrete packages rather than in a smooth flow" (p. 277), and (ii) systematically exhibiting "slack capacity relative to the demand of their owners" (ibid.), i.e., there exists a natural excess capacity of such goods. With the emergence of peer-to-peer trading platforms, such as AirBnB or Eloue, many consumers' preferences have been transformed to now favor access over ownership (Bardhi and Eckhardt 2012; Nielsen 2014; Belk 2014; Weber 2015). Sharing intermediaries have been able to solve problems of asymmetric information (Weber 2014), fuelling the growth of peer-to-peer exchanges (Einav et al. 2016).

\footnotetext{
${ }^{1}$ For instance, to restrict the use of a power drill to the original buyer, a manufacturer (or seller) could-if money is of no considerationrequire biometric recognition (e.g., via fingerprint or voice) to operate the machine.
} 
Sharing markets, by allowing for mutual insurance of consumers' ex post utilities derived from a combination of access to goods and the realization of ex ante uncertain needs, in the spirit of Arrow (1953), can at least partially correct for overcommitment (resp., undercommitment) of an individual's resources when (resp., not) owning a product. The economic rationality of collaborative consumption (Felson and Spaeth 1978; Botsman and Rogers 2010), when paired with sharing markets, has given rise to a sharing economy which is set to disrupt traditional modes of consumption and production. The present paper addresses the last point by analyzing a producer's (or retailer's) options to use the control of shareability for capturing secondary market rents in a peer-to-peer sharing economy.

\subsection{Outline}

The remainder of this paper is organized as follows: Sec. 2 introduces the model primitives and establishes the equilibrium choice behavior and market price in an overlapping-generations economy with sharing and nontrivial sharing tariff. Sec. 3 examines the firm's optimal pricing strategy, including conditions for a sharing shutdown, as well as several numerical examples. In Sec. 4. we discuss implications for product design, and Sec.5 concludes.

\section{Model}

As in Weber (2016a), we consider a sharing economy with overlapping generations of finitely lived consumers (or "agents"). Each consumer exists for two periods, termed the "early consumption phase" $\left(\mathcal{C}_{0}\right)$ and the "late consumption phase" $\left(\mathcal{C}_{1}\right)$, respectively. Because of the permanent coexistence of the two consumer generations, the sharing economy operates in steady state at times $t \in\{0,1, \ldots\}$. The number of consumers born in any given period $t$ is normalized to 1 , without any loss of generality. At the end of the following period, $t+1$, these consumers exit the economy. At any time, the total number of consumers in the sharing economy is 2 , as the sum of both coexisting consumer generations in their respective consumption phases.

The agents' preferences for consuming a given durable good (also referred to as "item" or "product") are heterogeneous in the subjective likelihood of need $\theta \in[0,1]$ and in the expected use value $\nu \in[0,1]$ conditional on a realized need. The durable good may be any rival product worth sharing 2 including subscription tickets, certain types of software (such as games), and physical devices, to name just a few. A consumer's "type" is therefore specified as a point $(\theta, \nu)$ in the square $\mathcal{Q}=[0,1] \times[0,1]$. For simplicity, we assume that the type distribution for any generation is uniform on $\mathcal{Q}$. Each consumer's type is persistent over his lifetime. The realizations of his need for the product are uncorrelated, and nothing can be learned from other consumers or his own consumption about his future demand.

\subsection{Dynamic Choice}

At the beginning of consumption phase $\mathcal{C}_{i}$, any given consumer of type $(\theta, \nu) \in \mathcal{Q}$ observes the realization $s_{i}$ of his random need state $\tilde{s}_{i} \in\{0,1\}$, which is distributed according to

$$
\operatorname{Prob}\left(\tilde{s}_{i}=1\right)=\theta,
$$

for all $i \in\{0,1\}$. Contingent on a realized high need state $\left(s_{i}=1\right)$, to get access to the product the agent can either purchase it from a retailer at the price $r>0$ or borrow it on a peer-to-peer market at the sharing price $p$. Regarding the latter, the right for a one-time use of the product can be traded (i.e., acquired or relinquished) on a sharing market where owners are asked to pay a transfer $\tau \geq 0$ to the firm for the authorization to rent the good to a peer at the (nonnegative) price $p<r 3^{3}$ Thus, ex ante before taking an ownership decision, a consumer considers the product both from the perspective of his benefits, as a function of his type (in terms of likelihood of need and contingent consumption value), his costs, and his opportunities ex post (in terms of $p, r, \tau$ ). The retail price $r$ and the sharing tariff $\tau$ are advertised with the firm's product offering, and $p$ is the (via rational expectations) correctly anticipated price in the sharing market.

Because the price $p$ for access in a sharing market cannot exceed the purchase price $r$, ownership decisions are taken only in the early consumption phase $\mathcal{C}_{0}$ as long as the sharing market is active. In the event of a sharing shutdown (see Sec. 3.2), high-value consumers who experience a need solely in their late consumption phase might still become owners. By contrast, with an enabled peer-to-peer economy consumers in their late consumption phase restrict attention to gaining and providing access to the good by taking the requisite borrowing and lending decisions on the sharing market. Given $p, r, \tau$ such that $0 \leq \tau \leq p \leq r$, we now analyze the agents' choice behavior in their two consumption phases using backward induction for a given generation of consumers; see Fig. 1 .

\subsubsection{Late Consumption Phase}

At the beginning of $\mathcal{C}_{1}$, a consumer of type $(\theta, \nu)$ observes the realization $s_{1} \in\{0,1\}$ of his need state $\tilde{s}_{1}$. If he is a non-owner, then he can either not consume

\footnotetext{
${ }^{2}$ The assumed rivalness in consumption implies that sharing the good reduces its availability to the owner.

${ }^{3} \mathrm{~A}$ situation where $p \geq r$ implies that the sharing market is inactive.
} 
the product at all or rent it on the sharing market at the price $p$. Given his lack of consideration about the future in his late consumption phase, renting dominates buying the product at the higher retail price $r>p$. For $p=r$, we assume that a consumer would prefer the residual benefits of ownership, thus leading to a shutdown of the sharing market, discussed in Sec. 3.2 below. Hence, a non-owner's state-dependent payoff can be written in the form

$$
U_{s_{1}}=\max \left\{0, \nu s_{1}-p\right\},
$$

i.e., all types with $\nu \in[p, 1]$ borrow in state $s_{1}=1$; otherwise, non-owners do nothing. Before delving into the details of the analysis, we introduce

$$
\hat{p} \triangleq p-\tau
$$

as the effective transaction price for lenders in the sharing market; it represents the net rent (or absolute markup) of a supplier in the sharing market. Indeed, if the consumer is an owner, then he has the option to keep the item for his own use or else pay $\tau$ to the firm to lend it out at the price $p$, with the state-dependent net payoff

$$
V_{s_{1}}=\max \left\{\nu s_{1}, \hat{p}\right\}
$$

i.e., all types with $\nu \in[0, \hat{p})$ lend in state $s_{1}=1$, and all types with $\nu \in(0,1]$ lend in state $s_{1}=0$; otherwise, owners take no action. The following result summarizes the state-contingent payoffs in $\mathcal{C}_{1}$.

Lemma 1. A type- $(\theta, \nu)$ agent's $\mathcal{C}_{1}$-payoffs are $U_{0}=$ $0, U_{1}=\max \{0, \nu-p\}$ as non-owner, and $V_{0}=$ $\max \{0, \hat{p}\}, V_{1}=\max \{\nu, \hat{p}\}$ as owner, respectively.

The payoff difference between owner and nonowner in the low-need state, $s_{1}=0$, is

$$
V_{0}-U_{0}=\max \{0, \hat{p}\}
$$

while in the high-need state, $s_{1}=1$, it is

$$
V_{1}-U_{1}= \begin{cases}\hat{p}, & \text { if } \nu<\hat{p} \\ \nu, & \text { if } \nu \in[\hat{p}, p] \\ p, & \text { if } \nu>p\end{cases}
$$

As long as the firm charges a positive sharing tariff, consumers with a high value of use may care distinctly more about ownership than those with a low value of use. Note also that for participants in the sharing market, the payoff is independent of the need state ${ }^{4}$

\subsubsection{Early Consumption Phase}

In $\mathcal{C}_{0}$, an individual of type $(\theta, \nu)$, who is in need state $s_{0}$, has the option to purchase the product from a retailer at the price $r$ to become an owner. In this case, the individual can use the item immediately, in that same consumption phase. Alternatively, the agent can rent the item on the sharing market at the price $p$. Note that at this early stage in his life, the agent forms an expectation about his future utility: $\bar{V}$ as owner or $\bar{U}$ as non-owner. All agents discount future payoffs at the common perperiod discount factor

$$
\delta \in(0,1]
$$

Choosing the best of his three alternatives (do nothing / borrow on the sharing market / buy from the retailer) an agent's discounted state-dependent total payoff is

$$
T_{s_{0}}=\max \left\{\delta \bar{U}, \nu s_{0}-p+\delta \bar{U}, \nu s_{0}-r+\delta \bar{V}\right\},
$$

where $\bar{U}=(1-\theta) U_{0}+\theta U_{1}$ and $\bar{V}=(1-\theta) V_{0}+\theta V_{1}$, and where $U_{i}, V_{i}$, for $i \in\{0,1\}$, are given by La. 1 Combining the first two decision options yields the total expected payoff of non-ownership,

$$
\left(s_{0}+\delta \theta\right) \max \{0, \nu-p\},
$$

which needs to be compared against the total expected payoff of ownership,

$$
\nu s_{0}-r+\delta(\hat{p}+\theta \max \{0, \nu-\hat{p}\}),
$$

separately for the agents' possible current need states.

In the low-need state, $s_{0}=0$, consider first individuals with contingent consumption values $\nu \geq p$ (and therefore also $\nu \geq \hat{p}$ ). Of those, agents with likelihood type

$$
\theta \geq \frac{r-\delta \hat{p}}{\delta \tau} \triangleq \vartheta_{01}
$$

would purchase the product. For contingent consumption values $\nu \in(\hat{p}, p)$, agents with likelihood type

$$
\theta \geq \frac{r-\delta \hat{p}}{\delta(\nu-\hat{p})} \triangleq \vartheta_{00}
$$

would like to buy the item. Lastly, no agent type with $\nu \leq \hat{p}$ would ever buy the item because they could not benefit from ownership more than by accessing the product as a borrower through the sharing market. For either threshold, $\vartheta_{00}$ or $\vartheta_{01}$, to not exceed 1 , i.e., for some types to be willing to buy the product, its retail price $r$ would have to be less than $\delta \min \{p, \nu\}$. But the latter is not possible, since $r>p$ is required for a functioning sharing market 5 This implies that no agent in the low-need state would ever purchase the product.

In the high-need state, $s_{0}=1$, consider first "highvalue" agents with values $\nu \geq p$. Acquisition of the product is then interesting for the likelihood types

$$
\theta \geq \max \left\{0, \frac{r-p-\delta \hat{p}}{\delta \tau}\right\} \triangleq \vartheta_{11} .
$$

\footnotetext{
${ }^{4}$ Indeed, $U_{0}=U_{1}$ for $\nu \in[p, 1]$ and $V_{0}=V_{1}$ for $\nu \in[0, \hat{p})$; owners with $\nu \in(\hat{p}, 1]$ lend only in the low-need state.

${ }^{5}$ Alternatively, for a type- $(\theta, \nu)$ agent to purchase, $r \leq \delta[\hat{p}+\theta \max \{0, \tau+\min \{0, \nu-p\}\}]$ needs to hold, contradicting $r>p$.
} 
Hence, there are some buyers (i.e., $\vartheta_{11} \leq 1$ ) iff (i.e., if and only if) the sharing price is bounded from below:

$$
p \geq r /(1+\delta) \triangleq p_{0}
$$

Conversely, all high-value agents buy (i.e., $\vartheta_{11}=0$ ) iff

$$
p \geq(r+\delta \tau) /(1+\delta) \triangleq p_{1} .
$$

To find ownership attractive, high-value agents need to anticipate a sufficiently high sharing price, in comparison to the retail price. Their aggregate demand for ownership is

$$
\Omega_{11}= \begin{cases}0, & \text { if } p<p_{0} \\ (1-p)\left(1-\vartheta_{11}^{2}\right) / 2, & \text { otherwise }\end{cases}
$$

Consider now "medium-value" agents with values between the lower price bound $p_{0}$ and the clearing price $p$ on the sharing market, so $\nu \in(\hat{p}, p)$. To them purchasing the item is attractive iff

$$
\theta \geq \max \left\{0, \frac{r-\nu-\delta \hat{p}}{\delta(\nu-\hat{p})}\right\} \triangleq \vartheta_{10}
$$

As before, some of the agents purchase (i.e., $\vartheta_{10} \leq 1$ ), as long as $p>p_{0}$, while all likelihood types are interested in ownership (i.e., $\vartheta_{10}=0$ ) iff $\nu \geq r-\delta \hat{p}$, which also requires that $p \geq p_{1}$. This leads to the aggregate demand for ownership from medium-value agents:

$$
\Omega_{10}= \begin{cases}0, & \text { if } p \leq p_{0} \\ \Omega_{10}^{A}, & \text { if } p_{0}<p<p_{1} \\ \Omega_{10}^{B}, & \text { if } p \geq p_{1}\end{cases}
$$

where we set

$$
\Omega_{10}^{A} \triangleq \int_{p_{0}}^{p}\left(\int_{\vartheta_{10}}^{1} \theta d \theta\right) d \nu=\int_{p_{0}}^{p} \frac{1-\vartheta_{10}^{2}}{2} d \nu
$$

and

$$
\begin{aligned}
\Omega_{10}^{B} & \triangleq \frac{p-(r-\delta \hat{p})}{2}+\int_{p_{0}}^{r-\delta \hat{p}} \frac{1-\vartheta_{10}^{2}}{2} d \nu \\
& =\frac{p-p_{0}}{2}-\frac{1}{2} \int_{p_{0}}^{r-\delta \hat{p}} \vartheta_{10}^{2} d \nu .
\end{aligned}
$$
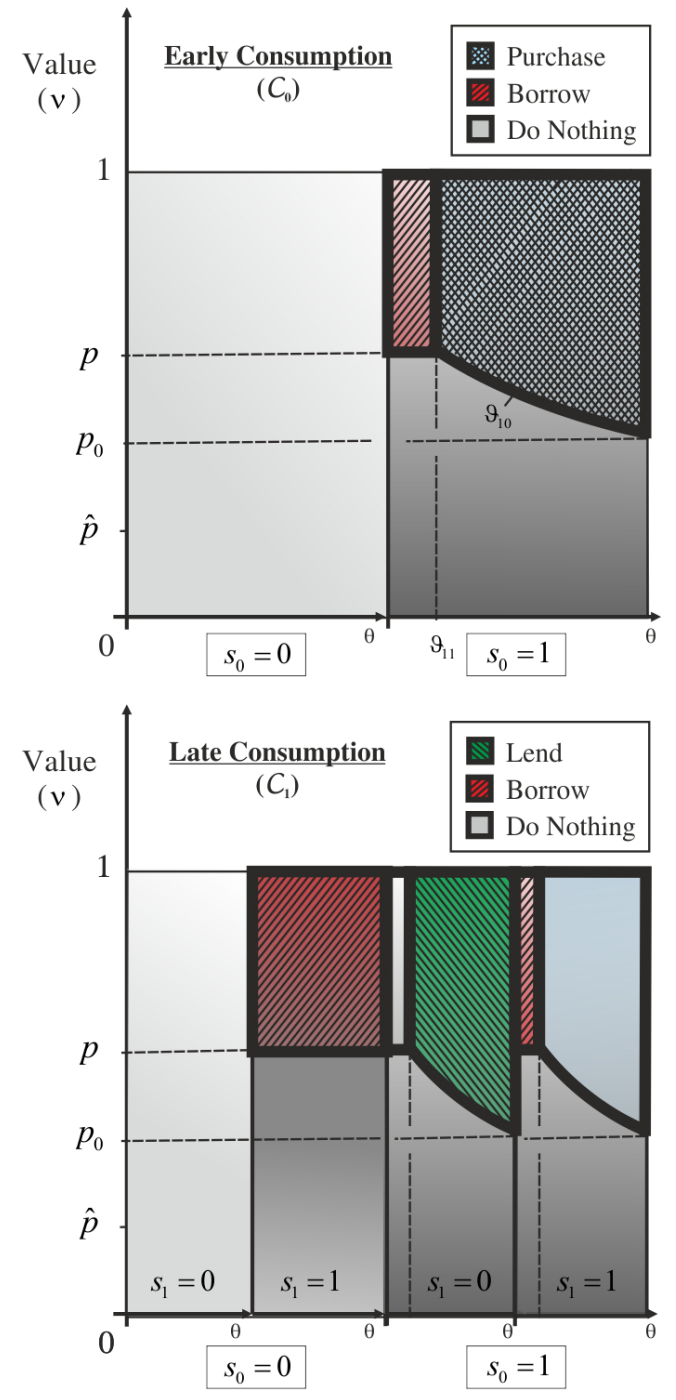

Figure 1: Dynamic choice behavior with sharing.

Taking into account that

$$
\begin{aligned}
\int_{p_{0}}^{p} \vartheta_{10}^{2} d \nu= & \frac{p_{0}-\hat{p}}{\delta^{2}}\left(\frac{p-p_{0}}{p_{0}-\hat{p}}+(1+\delta) \times\right. \\
& \left.\times\left[2 \ln \left(\frac{p_{0}-\hat{p}}{p-\hat{p}}\right)+(1+\delta) \frac{p-p_{0}}{p-\hat{p}}\right]\right),
\end{aligned}
$$

the preceding expressions can be computed explicitly ${ }^{6}$ It is interesting to observe that ownership by mediumvalue agents is an artefact produced by the existence of a positive sharing tariff as well as agents that care about the future 7

Combining the purchase decisions for high-value and medium-value agents in their early consumption phase yields the overall demand for the monopolist's goods in the presence of an active peer-to-peer market.

\footnotetext{
${ }^{6}$ Since $p=r-\delta \hat{p}$ implies $p-p_{0}=\delta\left(p_{0}-\hat{p}\right)$ and $p-\hat{p}=(1+\delta)\left(p_{0}-\hat{p}\right)$, one obtains $\Omega_{10}^{B}=\frac{p-p_{0}}{2}-\frac{p_{0}-\hat{p}}{\delta}\left(1+\frac{\delta}{2}-\frac{1+\delta}{\delta} \ln (1+\delta)\right)$.
}

${ }^{7}$ For $\delta \rightarrow 0^{+}$or $\tau \rightarrow 0^{+}$, one obtains that $\Omega_{10} \rightarrow 0$. 
Proposition 1. Given $p, \tau, r$ with $0 \leq \tau \leq r$, the aggregate demand for ownership in the (viable) sharing economy is $\Omega=\Omega_{10}+\Omega_{11}$.

This demand is based on the agents' choice behavior (see Fig. 1), which can be summarized as follows.

Lemma 2. In $\mathcal{C}_{0}$, a type- $(\theta, \nu)$ agent in the high-need state $s_{0}=1$ becomes an owner iff

$$
\theta \geq \frac{\max \{0, r-\min \{\nu, p\}-\delta \hat{p}\}}{\delta(\tau+\min \{0, \nu-p\})} \triangleq \theta_{0}(p, \nu) ;
$$

otherwise, if $\nu \geq p$, he borrows the item on the sharing market. In any other event, the agent does nothing.

The existence of a population that borrows in the early consumption phase (i.e., whether $\left.\theta_{0}(p, p)>0\right)$ depends on the price in the sharing market. For $p_{0}<p<$ $p_{1}$, agents with low likelihood types $\theta$ prefer gaining access to the product via sharing to an ownership commitment. In the absence of a sharing tariff, i.e., when $\tau=0$ the last condition becomes vacuous, and there is no sharing activity at all in the early consumption phase.

\subsection{Sharing Equilibrium}

Let $r>0$ be a given retail price and $\tau \in[0, p]$ be a sharing tariff. Assuming that the sharing market clears, the price $p$ in the sharing market must be such that demand for the shared product equals the supply ${ }^{8}$ The potential suppliers in the sharing market include all agents in their late consumption phase $\mathcal{C}_{1}$ who opted for ownership in their early consumption phase $\mathcal{C}_{0}$. Based on Prop. 1 and La. 2, the demand for ownership is

$$
\Omega=\int_{p_{0}}^{1}\left(\int_{\theta_{0}(p, \nu)}^{1} \theta d \theta\right) d \nu=\Omega_{10}+\Omega_{11},
$$

where $\nu=p_{0}$ is the lowest valuation of an agent (with $\theta=1$ ) purchasing the item. As implied by our discussion of the demand for ownership, the nature of the equilibrium depends on whether the sharing price is "high" (with $p>p_{1}$ ) or "moderate" (with $p_{0} \leq p \leq p_{1}$ ) 9 The following result helps focus the analysis of the sharing equilibrium, effectively excluding any high-price scenario in equilibrium; see App. A.1 for a proof.

Lemma 3. For any $(r, \tau) \geq 0$, a sharing market is active (i.e., it has positive trading volume) iff $r \in(0,1+\delta)$ and $\tau \leq p$. The clearing price $p$ of an active sharing market is moderate, i.e., it lies in the interval $\left[p_{0}, p_{1}\right]$.

The preceding result implies that in the special case without sharing tariff (for $\tau=0$ ), the market price is equal to $p_{0}$, i.e., $p=r /(1+\delta)$ as in Weber (2016a). It also allows us to concentrate on the interesting case where $p \in\left[p_{0}, p_{1}\right]$.

\subsubsection{Supply}

The supply in the sharing market consists of owners who are happy to lend when they find themselves in a lowneed state $\left(s_{1}=0\right)$. In a high-need state $\left(s_{1}=1\right)$, owners with values $\nu<\hat{p}$ (who are a priori willing to lend) do not exist, as owners' lowest value is $p_{0} \geq \hat{p}$. Thus, the sharing supply becomes

$$
S=\int_{p_{0}}^{1}\left(\int_{\theta_{0}(p, \nu)}^{1}(1-\theta) \theta d \theta\right) d \nu
$$

Aggregating owners with $\nu \in[p, 1]$ yields the supply

$$
\begin{aligned}
S_{11} & =\int_{p}^{1}\left(\int_{\vartheta_{11}}^{1}(1-\theta) \theta d \theta\right) d \nu \\
& =(1-p)\left(\frac{1}{6}-\frac{\vartheta_{11}^{2}}{2}+\frac{\vartheta_{11}^{3}}{3}\right)
\end{aligned}
$$

while owners with $\nu \in\left[p_{0}, p\right]$ constitute a supply of

$$
\begin{aligned}
S_{10} & =\int_{p_{0}}^{p}\left(\int_{\vartheta_{10}}^{1}(1-\theta) \theta d \theta\right) d \nu \\
& = \begin{cases}0, & \text { if } p \leq p_{0} \\
S_{10}^{A}, & \text { if } p_{0}<p<p_{1} \\
S_{10}^{B}, & \text { if } p \geq p_{1}\end{cases}
\end{aligned}
$$

where, using the abbreviation $\lambda \triangleq\left(p_{0}-\hat{p}\right) / \tau 10$

$$
\begin{aligned}
S_{10}^{A}= & \frac{p-p_{0}}{6}-\int_{p_{0}}^{p}\left(\frac{\vartheta_{10}^{2}}{2}-\frac{\vartheta_{10}^{3}}{3}\right) d \nu \\
= & \frac{(1-\lambda) \tau}{6}\left(1-\frac{3}{\delta^{2}}-\frac{2}{\delta^{3}}\right)-\frac{(1+\delta)^{2} \lambda \tau}{\delta^{3}} \times \\
& \left(\ln (\lambda)+(1-\lambda) \frac{5+2 \delta-(1+\delta) \lambda}{6}\right)
\end{aligned}
$$

This yields the sharing supply, $S=S_{10}+S_{11}$, for any combination of retail price and sharing tariff which allow for an active (i.e., positive-trading-volume) peer-topeer exchange in the secondary market.

Lemma 4. For any $(r, \tau) \geq 0$ with active sharing market, the sharing supply is $S=S_{10}^{A}+S_{11}$.

The sharing supply collapses whenever the sharing tariff $\tau$ exceeds the equilibrium price $p$ in the market because this would entail a negative absolute markup $\hat{p}$. Naturally, the equilibrium price also depends on $\tau$, so that checking whether $\tau$ exceeds $p$ in equilibrium involves solving the corresponding fixed-point problem, which is discussed below.

\footnotetext{
${ }^{8}$ In practice, there may be supply-demand imbalances (Weber 2014a,b; Razeghian and Weber 2015).

${ }^{9}$ For "low" sharing prices (with $p<p_{0}$ ), the demand for ownership vanishes, thus also disabling any peer-to-peer aftermarket.

${ }^{10}$ Further analytical details (e.g., on obtaining $S_{10}^{B}$, which by La. 3 is not relevant in equilibrium) are provided in App. A.
} 


\subsubsection{Demand}

Non-owners like to access a product on the sharing market whenever they are in a high-need state (in either consumption phase) and their contingent use value exceeds the market price (so $\nu \geq p$ ). The corresponding sharing demand from the mature generation in $\mathcal{C}_{1}$ amounts to

$$
D_{1}=\int_{p}^{1}\left(\int_{0}^{1}(1-\theta) \theta d \theta+\int_{0}^{\theta_{0}(p, \nu)} \theta^{2} d \theta\right) d \nu,
$$

while for the early generation in $\mathcal{C}_{0}$ it is

$$
D_{0}=\int_{p}^{1}\left(\int_{0}^{\theta_{0}(p, \nu)} \theta d \theta\right) d \nu .
$$

Hence, for $p_{0}<p<p_{1}$, we obtain

$$
D=D_{0}+D_{1}=(1-p)\left(\frac{1}{6}+\frac{\vartheta_{11}^{2}}{2}+\frac{\vartheta_{11}^{3}}{3}\right) .
$$

\subsubsection{Market Clearing}

For a sharing price $p \in\left[p_{0}, p_{1}\right]$, supply equals demand ( $S=D$; i.e., lenders $=$ borrowers in Fig. 1 ) iff

$$
S_{10}^{A}=(1-p) \vartheta_{11}^{2} .
$$

Since, by definition, the type threshold $\vartheta_{11}$ is

$$
\vartheta_{11}=1-\frac{(1+\delta)(1-\lambda)}{\delta}=\frac{(1+\delta) \lambda-1}{\delta},
$$

the sharing price becomes a function of $\lambda$ and $\tau$ :

$$
p=1-\left(\frac{\delta}{(1+\delta) \lambda-1}\right)^{2} S_{10}^{A} \triangleq h(\lambda, \tau) .
$$

From this we can determine $\lambda=\varphi(r, \tau)$ by solving a fixed-point problem (using the definition of $\lambda$ ):

$$
\lambda=1-\frac{1}{\tau}\left(h(\lambda, \tau)-\frac{r}{1+\delta}\right) \triangleq \varphi(r, \tau) ;
$$

this finally yields the equilibrium sharing price $p^{*}$ as a function of $r$ and $\tau 11$

Proposition 2. For any $(r, \tau) \geq 0$ with active sharing market, the equilibrium sharing price $p^{*}=$ $h(\varphi(r, \tau), \tau)$ lies in the interval $\left[p_{0}, p_{1}\right]$.

A straightforward analysis reveals a monotone dependence of the equilibrium sharing price on the monopolist's choice variables.

Lemma 5. Provided there is an active sharing market, the equilibrium sharing price $p^{*}$ is increasing in $(r, \tau) \geq 0$.
By increasing the sharing tariff beyond the equilibrium market price, the monopolist can effectively disable the sharing market (i.e., induce a "sharing shutdown") because in that case the absolute markup $\hat{p}$ becomes negative, thus negating any economic incentives peers may have had to make their goods available for collaborative consumption. The question of which precise bound this implies on the sharing tariff is answered by solving the fixed-point problem $\tau=p^{*}(r, \tau)$, discussed in the next section because it is central to the solution of the monopolist's profit-maximization problem.

\section{Optimal Pricing}

\subsection{Active Sharing}

With an active peer-to-peer market, the firm has two sources of revenue: the sales of the product and the rent from the sharing tariff on sharing transactions. Thus, its profits are of the form

$$
\Pi=(r-c) \Omega+\tau D,
$$

subject to the equilibrium sharing price $p=p^{*}(r, \tau)$ in Prop. 2 As already pointed out, for the sharing market to stay liquid, the sharing tariff $\tau$ cannot exceed the equilibrium sharing price. This implies a discontinuity at the boundary $\tau=p^{*}(r, \tau)$ in the monopolist's profit function. We also note that for a zero sharing tariff, we can revert to simpler optimality conditions to obtain an optimal retail price ${ }^{12}$ The power of the sharing tariff is that it allows the monopolist to syphon off surplus from the sharing market at a point where established owners (in $\mathcal{C}_{1}$ ) have observed their need states. For any given sharing tariff $\tau$, the firm's profit has an interior optimum $r^{*}(\tau) \in(c, 1+\delta)$ because a price at the boundary would lead to zero profits. Thus, $r^{*}(\tau)$ can be determined from a (modified) monopoly pricing rule (see, e.g., Tirole 1988, p. 66).

Proposition 3. In an active sharing market, for a given sharing tariff $\tau \in\left[0, p^{*}\right]$, the optimal retail price $r^{*}(\tau)$ satisfies the following inverse-elasticity rule for sharing:

$$
\frac{r-c}{r}=\frac{1+(\tau / r)\left(D^{*} / \Omega^{*}\right) \hat{\varepsilon}}{\varepsilon},
$$

where $\varepsilon \triangleq-r \Omega_{r}^{*} / \Omega^{*}$ is the own-price elasticity of the equilibrium demand for ownership $\left(\Omega^{*}(r, \tau) \triangleq\right.$ $\left.\Omega\left(p^{*}(r, \tau), r, \tau\right)\right)$ and $\hat{\varepsilon} \triangleq r D_{r}^{*} / D^{*}$ is the cross-price elasticity of the equilibrium demand in the sharing market $\left(D^{*}(r, \tau) \triangleq D\left(p^{*}(r, \tau), r, \tau\right)\right)$ with respect to the retail price ${ }^{13}$

\footnotetext{
${ }^{11}$ Although a closed-form solution is available, it has been omitted here due to its complexity.

${ }^{12}$ For $\tau=0$, the optimal retail price is $r_{0}^{*}=(1+\delta+c) / 2$ resulting in the profit $\Pi_{0}^{*}=(1+\delta-c)^{2} /(8(1+\delta))$; see Weber (2016a).

${ }^{13}$ The subscripts denote partial derivatives.
} 

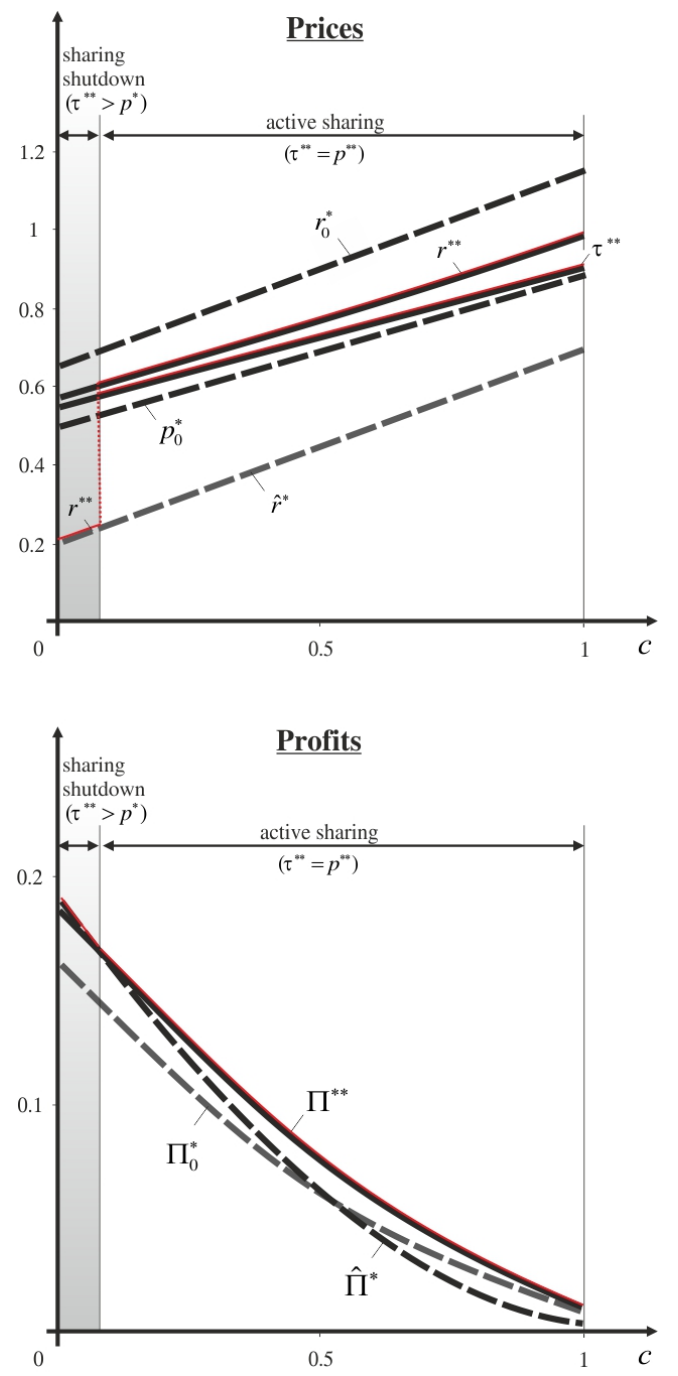

Figure 2: Equilibrium prices and profits $(\delta=0.3)$.

Because an active peer-to-peer market requires the sharing price to be less than the retail price, a forteriori the sharing tariff is also less than the retail price. In addition, the transaction volume in the sharing market is bounded from above by the number of goods owned in the economy (which is given by the equilibrium demand for ownership in our model). Hence, the factor $(\tau / r)\left(D^{*} / \Omega^{*}\right)<1$, limiting the influence of the crossprice elasticity $(\hat{\varepsilon})$ of the sharing demand. The latter becomes irrelevant for $\tau=0$ when the optimality condition specializes to the standard monopoly pricing rule for sharing markets (see footnote 12 ).

Substituting the retail price $r^{*}(\tau)$, the firm's equilibrium profit depends only on $\tau$ and needs to be maximized on the interval $[0, \bar{\tau}]$, where the upper bound for the sharing tariff is determined (implicitly) by the fixedpoint problem

$$
\bar{\tau}=p^{*}\left(r^{*}(\bar{\tau}), \bar{\tau}\right) .
$$

Necessary and sufficient optimality conditions for glob- ally maximizing any continuously differentiable function on an interval (here $[0, \bar{\tau}]$ ) have been recently developed by Weber (2016b).

Proposition 4. The monopolist's optimal product offer$\operatorname{ing}\left(r^{* *}, \tau^{* *}\right)$ is such that $r^{* *}=r^{*}\left(\tau^{*}\right)$, and $\tau^{* *}$ solves

$$
\max _{\tau \in[0, \bar{\tau}]}\left\{\left(r^{*}(\tau)-c\right) \Omega^{*}\left(r^{*}(\tau), \tau\right)+\tau D^{*}\left(r^{*}(\tau), \tau\right)\right\} .
$$

The preceding result implies the equilibrium price on the (by construction active) sharing market:

$$
p^{* *}=p^{*}\left(r^{* *}, \tau^{* *}\right) \in\left[p_{0}, p_{1}\right] .
$$

For sufficiently small production cost it has been recently shown that without sharing tariff, i.e., for $\tau=0$ and $c \geq 0$ small, the firm prefers no sharing to sharing (Weber 2016a), even when it has control over a product's durability (Razeghian and Weber 2016). Accordingly, we examine next the possibility of deliberate sabotage of the sharing market by the monopolist's leveraging its control of shareability.

\subsection{Sharing Shutdown}

In the event the sharing tariff is too high (so it would exceed the equilibrium sharing price), the sharing market becomes illiquid and breaks down. The peer-to-peer market is therefore not accessible, and consumers need to make "isolated" consumption decisions. This situation reverts to the case without sharing examined by Weber (2016a). One can backward-induct ownership decisions over the agents' lifecycles and derive the ownership demand $\hat{\Omega}_{i}$ in $\mathcal{C}_{i}$ for $i \in\{0,1\}$ :

$$
\hat{\Omega}_{0}=\frac{(r-\delta)^{2}-1}{2 \delta^{2}}-\left(\frac{\ln (r)-\ln (1+\delta)}{\delta}\right) \frac{r}{\delta},
$$

and

$$
\hat{\Omega}_{1}=\max \left\{0, \frac{1-r}{6}\right\} .
$$

The total demand for ownership in the absence of a sharing market is

$$
\hat{\Omega}=\hat{\Omega}_{0}+\hat{\Omega}_{1},
$$

for any given retail price $r \in(0,1+\delta)$. Correspondingly, the firm's no-sharing profit is

$$
\hat{\Pi}=(r-c) \hat{\Omega} .
$$

For sufficiently small cost, $c \in[0,1 / 2]$, the optimal retail price without sharing can be obtained in closed form,

$$
\hat{r}^{*}=\frac{1}{3 \rho}+\frac{c}{2},
$$

resulting in the optimal no-sharing profit

$$
\hat{\Pi}^{*}=\frac{(2-3 \rho c)^{2}}{36 \rho},
$$


where $\rho \triangleq \frac{1}{6}+\frac{1}{\delta}\left(1-\frac{\ln (1+\delta)}{\delta}\right) \in[(7 / 6)-\ln (2), 2 / 3]$ is a decreasing function of $\delta \in(0,1]$.
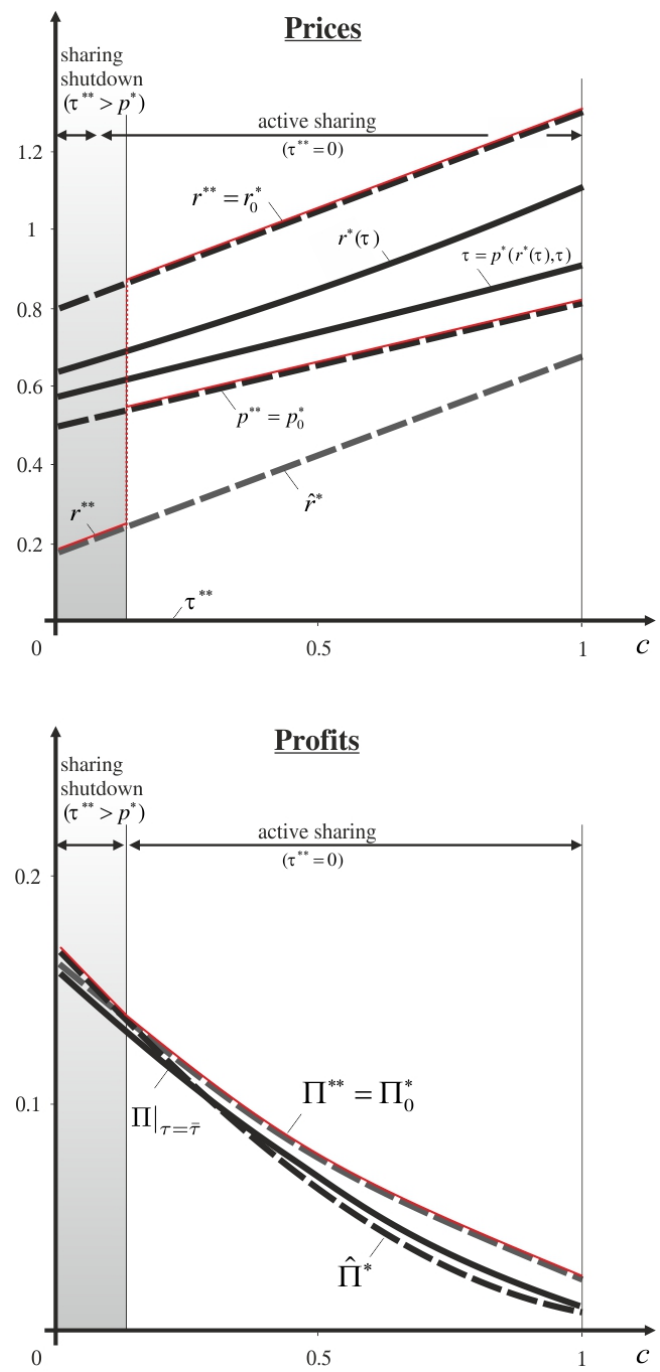

Figure 3: Equilibrium prices and profits $(\delta=0.6)$.

\subsection{Implementation}

Figs. 2 and 3 show the optimal profits and prices for $\delta=0.3$ and $\delta=0.6$. In the absence of shareability control (i.e., for $\tau=0$ ), the company would prefer no sharing to sharing. A positive sharing tariff $\tau \in(0, \bar{\tau}]$ can improve the monopolist's profit for impatient (low$\delta$ ) customers over sharing with $\tau=0$ (i.e., without tariff) and over no sharing. In this situation, the extra revenue from the secondary market provides a positive incentive for the firm to support collaborative consumption of its products. By contrast, for impatient (high$\delta$ ) customers and high-cost products the firm is best off with sharing but without a sharing tariff. For low-cost products, $\tau \in(0, \bar{\tau}]$ is still not optimal, but the firm can use an excessive sharing tariff to trigger a sharing shutdown, using its control over the shareability of its products to disable the peer-to-peer secondary market.

\section{Implications for Product Design}

Using the sharing tariff $\tau$ as an additional price instrument, the monopolist has the option to extract contingent rents from low-need owners or else to disable the sharing market altogether. As shown in Fig. 2, the benefits from extracting "shareability rents" can be significant, possibly justifying an investment in proprietary technology that enables aftermarket control ${ }^{14}$ The firm would want to invest in technologies that ensure its control over the owner's sharing activities. Thus, to seek the extra rent a monopolist would want to make a one-time investment $I$ of (at most) the net present value of the perpetuity of the excess profit. On the other hand, any per-period cost $\gamma$ (e.g., monitoring, accounting, and administration) related to gaining and maintaining shareability control would need to be compared to the perperiod benefit, so that in total

$$
I+\frac{\gamma}{1-\delta} \leq \frac{\Pi^{* *}-\Pi_{0}^{*}}{1-\delta},
$$

for all $\delta \in(0,1){ }^{15}$ In order to fully appropriate the shareability rent the firm may need to run its own secondary sharing market. Yet, because of the critical mass already attained by existing sharing platforms, it may need to limit its take so as to accommodate the commissions charged by third-party intermediaries and still leave sufficient incentives for owners on the table to share their products.

\section{Conclusion}

Controlling the shareability of products allows a company to align its pricing instruments closer with consumer needs, as they arise. Depending on its production cost and the nature of consumers, it can be optimal to omit a sharing tariff ( $\tau=0$ ), or else to use $\tau>0$ in order to either extract a shareability rent from peer-topeer transactions $(\tau=p)$ or to shut down the sharing market altogether $(\tau>p)$ removing owners' economic incentive to offer their products to peers. The last option transposes the manufacturer to a situation without sharing that tends to maximize profits for low-cost products and sufficiently patient (non-myopic) customers. In-

\footnotetext{
${ }^{14}$ As usual "rent seeking," the expected benefit from controlling shareability justifies spending some (or even all) of it in order to attain it (Tullock 1980), resulting possibly in the dissipation of the entire shareability rent.

${ }^{15}$ For $\delta=1$, i.e., when there is no discounting, then the firm would want to spend whatever it takes $(I \leq \infty)$ to gain control of the shareability of its products, as long as it yields a positive per-period benefit (net of the per-period $\operatorname{cost} \gamma$ ).
} 
deed, without sharing, the product price is lower and demand for ownership is relatively high.

The second option, optimal for relatively impatient customers, is for the manufacturer to charge a sharing tariff $\tau=p$ that extracts all rent from the secondary market without shutting it down altogether. Because this option relies, in our theoretical model, on the willingness of owners to share in a low-need state even when obtaining a zero absolute markup, the recommendation is not exactly robust with respect to even small misperceptions or perturbations of the model. The conclusion in this regime is more of an indication that a positive sharing tariff-while maintaining a liquid sharing market— can be profitable for the firm (see Fig. 2), despite the fact that the primary effect of the positive sharing tariff is to reduce demand for ownership and decrease the retail price ${ }^{16}$ Indeed, its secondary effect is to increase the sharing price narrowing the gap between retail price $r$ (i.e., the price on the primary market) and the sharing price $p$ (i.e., the price on the secondary market). Finally, for high-cost products and sufficiently patient products it is interesting to note that the firm's optimal sharing tariff $\tau$ is zero (see Fig. 3): even though it has full control over the shareability of its products it is in its own best interest to not exercise it at all.

\section{Appendix A: Analytical Details}

\section{A.1 Moderate Sharing Price}

To prove that the sharing price $p$ needs to lie in the interval $\left[p_{0}, p_{1}\right]$, assume that $p>p_{1}$, which implies, by virtue of $\Omega_{10}=\Omega_{10}^{B}$, the demand of ownership

$$
\Omega=\frac{1}{2}\left(1-(1-p) \vartheta_{11}^{2}-p_{0}-\beta\left(p_{0}-\hat{p}\right)\right),
$$

with the constant coefficient

$$
\beta \triangleq \frac{2}{\delta}\left(1+\frac{\delta}{2}-\frac{1+\delta}{\delta} \ln (1+\delta)\right) \in(0,3-4 \ln (2))
$$

The sharing supply by medium-value owners is

$$
S_{10}^{B}=\frac{p-p_{0}}{6}-\int_{p_{0}}^{r-\delta \hat{p}}\left(\frac{\vartheta_{10}^{2}}{2}-\frac{\vartheta_{10}^{3}}{3}\right) d \nu
$$

To compute the relevant integrals, it is convenient to set

$$
\Lambda \triangleq \frac{r-(1+\delta) \hat{p}}{\nu-\hat{p}}=(1+\delta)\left(\frac{p_{0}-\hat{p}}{\nu-\hat{p}}\right),
$$

whence (omitting the constants in indefinite integrals):

$$
\int \frac{\vartheta_{10}^{2}}{2} d \nu=\frac{\nu}{2 \delta^{2}}-\frac{\nu-\hat{p}}{\delta^{2}}\left(\frac{\Lambda^{2}}{2}+\Lambda \ln (\nu-\hat{p})\right),
$$

and

$$
\int \frac{\vartheta_{10}^{3}}{3} d \nu=-\frac{\nu}{3 \delta^{3}}+\frac{\nu-\hat{p}}{\delta^{3}}\left(\Lambda^{2}-\frac{\Lambda^{3}}{6}+\Lambda \ln (\nu-\hat{p})\right) .
$$

${ }^{16}$ In practice, a sharing tariff strictly below the sharing price (leaving a "robustness margin") may help avoid inadvertent sharing shutdowns.
Note that $\left.\Lambda\right|_{\nu=r-\delta \hat{p}}=1$ and $\left.\Lambda\right|_{\nu=p_{0}}=1+\delta$. The two preceding integrals, taken between the bounds of $p_{0}$ and $r-\delta \hat{p}$, evaluate to

$$
\frac{p_{0}-\hat{p}}{\delta}\left[1+\frac{\delta}{2}-(1+\delta) \frac{\ln (1+\delta)}{\delta}\right]
$$

and

$$
\frac{p_{0}-\hat{p}}{\delta^{2}}\left[-1-\frac{\delta}{2}+\frac{\delta^{2}}{6}+(1+\delta) \frac{\ln (1+\delta)}{\delta}\right],
$$

respectively. We therefore obtain

$$
S_{10}^{B}=\alpha\left(p-p_{0}\right)-\left(\alpha-\frac{1}{6}\right) \tau=\frac{\tau}{6}-\alpha\left(p_{0}-\hat{p}\right),
$$

for $p \geq p_{1}$, where

$$
\alpha \triangleq \frac{1+\delta}{\delta^{2}}\left[1+\frac{\delta}{2}-(1+\delta) \frac{\ln (1+\delta)}{\delta}\right] .
$$

It is $1 / 6<\alpha \leq 3-4 \ln (2) \approx 0.2274$ for $\delta \in(0,1]$. Combining this with the earlier findings yields the sharing supply:

$$
S=\frac{1-p_{0}}{6}-\hat{\alpha}\left(p_{0}-\hat{p}\right)=\frac{1}{6}-\frac{\alpha r}{1+\delta}+\hat{\alpha}(p-\tau),
$$

where $\hat{\alpha} \triangleq \alpha-(1 / 6)>0$ and $p \geq p_{1}$. The supply in the sharing market is decreasing in the retail price and the sharing tariff, and it is increasing in the sharing price. On the other side of the market, the demand in a high-price regime stems exclusively from agents with need-state realizations $s_{0}=0$ and $s_{1}=1$, so

$$
D=\int_{p}^{1}\left(\int_{0}^{1}(1-\theta) \theta d \theta\right) d \nu=\frac{1-p}{6} .
$$

Equating supply and demand $(S=D)$ implies a linear dependence of the market price on retail price and sharing tariff:

$$
p^{*}(r, \tau)=\frac{r}{1+\delta}+\left(\frac{\hat{\alpha}}{\alpha}\right) \tau=p_{0}+\left(\frac{\hat{\alpha}}{\alpha}\right) \tau,
$$

where $\hat{\alpha} \triangleq \alpha-(1 / 6)>0$. However, the assumption $p^{*}(r, \tau)>p_{1}$ implies that $(\hat{\alpha} / \alpha)>\delta /(1+\delta)$, which does not hold for any $\delta \in$ $(0,1]$. Thus, by contradiction of the counterfactual we obtain that the clearing price of an active sharing market must be "moderate" in the sense that it lies in the interval $\left[p_{0}, p_{1}\right]$.

\section{A.2 Supply at a Moderate Sharing Price}

At the moderate sharing price $p \in\left[p_{0}, p_{1}\right]$, the supply from mediumvalue owners is

$$
S_{10}^{A}=\frac{p-p_{0}}{6}-\int_{p_{0}}^{p}\left(\frac{\vartheta_{10}^{2}}{2}-\frac{\vartheta_{10}^{3}}{3}\right) d \nu
$$

as noted in the main text. Using the expressions for the indefinite in tegrals in App. A.1, between the bounds $p_{0}$ and $p$, one obtains

$$
\begin{aligned}
\int_{p_{0}}^{p} \frac{\vartheta_{10}^{2}}{2} d \nu= & \frac{p-p_{0}}{\delta^{2}}\left[\frac{1}{2}+\frac{(1+\delta)^{2}}{2}\left(\frac{p_{0}-\hat{p}}{\tau}\right)\right. \\
& \left.+(1+\delta)\left(\frac{p_{0}-\hat{p}}{p-p_{0}}\right) \ln \left(\frac{p_{0}-\hat{p}}{\tau}\right)\right] \\
= & \frac{\tau}{\delta^{2}}\left[\frac{1-\lambda}{2}+(1+\delta) \lambda \ln (\lambda)\right. \\
& \left.+\frac{(1+\delta)^{2}}{2} \lambda(1-\lambda)\right]
\end{aligned}
$$

and

$$
\begin{aligned}
\int_{p_{0}}^{p} \frac{\vartheta_{10}^{3}}{3} d \nu= & \frac{\tau}{\delta^{3}}\left[-\frac{1-\lambda}{3}-(1+\delta) \lambda \ln (\lambda)\right. \\
& \left.-(1+\delta)^{2} \lambda(1-\lambda)\left(1-\frac{1+\delta}{6}(1+\lambda)\right)\right],
\end{aligned}
$$

where we recall that $\lambda=\left(p_{0}-\hat{p}\right) / \tau$. This implies the expression for the supply $S_{10}^{A}$ given in the main text. 


\section{References}

[1] AdAms, W., BRock, J.W. (1982) "Integrated Monopoly and Market Power: System Selling, Compatibility Standards, and Market Control," Quarterly Review of Economics and Business, Vol. 22, No. 4, pp. 29-42.

[2] ARrow, K.J. (1953) "Le rôle des valeurs boursières pour la répartition la meilleure des risques," Économetrie, Centre National de la Recherche Scientifique, Vol. 11, pp. 41-47. [Reprinted: "The Role of Securities in the Optimal Allocation of Risk Bearing," Review of Economic Studies, Vol. 31, No. 2, pp. 91-96, 1964.]

[3] Bardhi, F., EcKhardt, G.M. (2012) "AccessBased Consumption: The Case of Car Sharing," Journal of Consumer Research, Vol. 39, No. 4, pp. 881-898.

[4] BelK, R. (2014). "You Are What You Can Access: Sharing and Collaborative Consumption Online," Journal of Business Research, Vol. 67, No. 8, pp. 1595-1600.

[5] BenKLER, Y. (2004) "Sharing Nicely: On Shareable Goods and the Emergence of Sharing as a Modality of Economic Production," Yale Law Journal, Vol. 114, No. 2, pp. 273-358.

[6] Botsman, R., Rogers, R. (2010) What's Mine Is Yours: How Collaborative Consumption Is Changing the Way We Live, HarperCollins, London, UK.

[7] Carlton, D.W., Waldman, M. (2002) "The Strategic Use of Tying to Preserve and Create Market Power in Evolving Industries," RAND Journal of Economics, Vol. 33, No. 2, pp. 194-220.

[8] Einav, L., Farronato, C., Levin, J. (2016) "Peer-to-Peer Markets, Annual Review in Economics, Vol. 8, Forthcoming.

[9] Farrell, J., Saloner, G. (1992) "Converters, Compatibility, and the Control of Interfaces," Journal of Industrial Economics, Vol. 40, No. 1, pp. 9-35.
[10] Felson, M., Spaeth, J.L. (1978) “Community Structure and Collaborative Consumption: A Routine Activity Approach," American Behavioral Scientist, Vol. 21, No. 4, pp. 614-624.

[11] NiELSEN (2014) "Is Sharing the New Buying?" Nielsen Company, New York, NY, May 28.

[12] Razeghian, M., Weber, T.A. (2015) “To Share or Not to Share: Adjustment Dynamics in Sharing Markets," Working Paper, École Polytechnique Fédérale de Lausanne, Lausanne, Switzerland.

[13] Razeghian, M., Weber, T.A. (2016) "The Impact of Sharing Markets on Product Durability," Working Paper, École Polytechnique Fédérale de Lausanne, Lausanne, Switzerland.

[14] Schulz, L. (2015) "The Economics of Aftermarkets," Journal of European Competition Law and Practice, Vol. 6, No. 2, pp. 123-128.

[15] Tirole, J. (1988) The Theory of Industrial Organization, MIT Press, Cambridge, MA.

[16] Tullock, G. (1980) "Efficient Rent Seeking," in: Buchanan, J.M., Tollison, R.D., Tullock, G. (eds.) Toward a Theory of the Rent-Seeking Society, Texas A\&M University Press, College Station, TX, pp. 97-112.

[17] Weber, T.A. (2014) "Intermediation in a Sharing Economy: Insurance, Moral Hazard, and Rent Extraction," Journal of Management Information Systems, Vol. 31, No. 3, pp. 35-71.

[18] Weber, T.A. (2015) "The Question of Ownership in a Sharing Economy," Proceedings of the 48th Annual Hawaii International Conference on System Sciences (HICSS), IEEE Computer Society, Washington, DC, pp. 4874-4883.

[19] Weber, T.A. (2016a) "Product Pricing in a Peerto-Peer Economy," Journal of Management Information Systems, Forthcoming.

[20] Weber, T.A. (2016b) "Global Optimization on an Interval," Journal of Optimization Theory and Applications, Forthcoming. 